\title{
Root extrusion, a practical solution in complicated crown-root incisor fractures
}

\author{
G. J. Brown,' and R. R.Welbury, ${ }^{2}$
}

\begin{abstract}
Implants and fixed and removeable prostheses are very successful in replacing missing units but their cost can be inhibitory to a number of patients. In addition fixed and removeable prostheses can be destructive to sound abutment teeth and can result in damage to dental and soft tissue. This report describes the restoration of a tooth with a complicated incisor crown-root fracture that extended below both the gingival cuff and the alveolar crest, by using remaining tooth tissue. The restoration was completed after root extrusion with a cast post, diaphragm and core, and porcelain crown.
\end{abstract}

$\mathrm{O}$ blique crown fractures involving enamel, dentine and pulp that extend below both the gingival margin and the alveolar bone are difficult to restore. Attempts to expose the fracture line by alveolar re-contouring alone may compromise the functional root length. In addition, poor aesthetics may result from any attempt to re-contour the labial tissues with simple or complex periodontal techniques.

It is generally accepted that coronal restorations in the anterior region require a residual root length at least equivalent to that of the clinical crown for long-term stability. Extrusion planning should take this into account.

Orthodontic extrusion of roots was described more than 20 years ago by Heithersay ${ }^{1}$ Ingber ${ }^{2,3}$ and Simon. ${ }^{4}$ Cook reported that ideal rapid extrusion of a single root requires forces of the magnitude of $0.7 \mathrm{~N}$ to $1.5 \mathrm{~N} .^{5}$ Movements of between $4-6 \mathrm{~mm}$ over $6-8$ weeks can be achieved using these light forces. Excessive forces can lead to pain, failure of the tooth to move, root damage, tilting of the abutment teeth and subsequent impaction of the root being extruded. Rapid extrusion is necessary to prevent movement of the gin-

\footnotetext{
$1^{*}$ Senior Dental Officer, Eston Health Clinic, Fabian Road, Eston, Middlesbrough TS6 9AP; ${ }^{2}$ Consultant in Paediatric Dentistry, Dental Hospital and School, Richardson Road, Newcastle upon Tyne NE2 4AZ ${ }^{*}$ Correspondence to: $M r$ Gavin Brown REFEREED PAPER

Received 09.05.00; Accepted 26.07.00

(C) British Dental Journal 2000; 189: 477-478
}

gival collar and alveolar bone with the root. Practically there is always some movement of these tissues but considerably less than if the extrusion was completed with lesser forces at a slower rate.

The most frequently described technique follows root canal obturation and uses a post made from orthodontic wire with a j-hook at its coronal end. The straight part of the post is ridged with a diamond bur in an air rotor to aid retention and cemented into the canal with a firm temporary cement (IRM). Traction down the long axis of the root is provided by stretching an elastic

$$
\begin{aligned}
& \text { In brief } \\
& \text { A simple technique for restoration of a } \\
& \text { tooth with a complicated incisor } \\
& \text { crown-root fracture which: } \\
& \text { - requires no specialist training } \\
& \text { - involves minimal capital cost, and } \\
& \text { - allows for natural tooth preservation. }
\end{aligned}
$$

band from the j-hook over a wire cemented to the incisal edges of the abutment teeth and then back to the j-hook. The wire attached to the abutment teeth should ideally be bent so that traction is through the long axis of the root and not labially. A fibrotomy, prior to starting treatment and also following treatment has been advocated. Re-organisation of the periodontal fibres requires around four weeks, ${ }^{6}$ and retention of the root in its new position is advisable for at least this period prior to

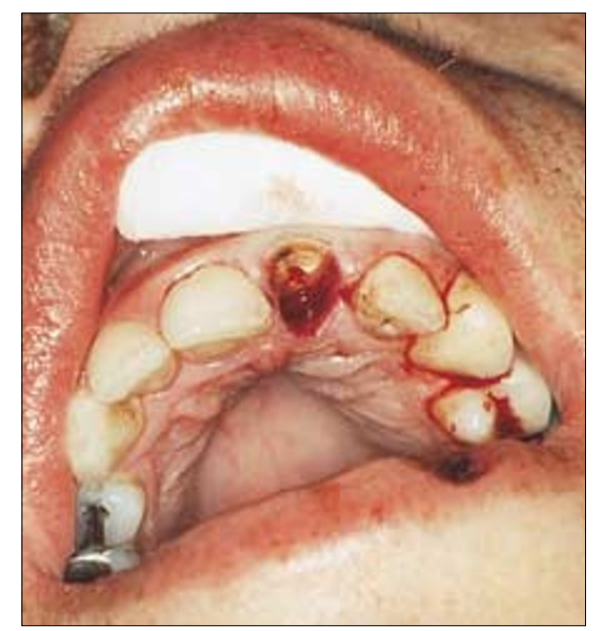

Fig. I Appearance of the root face after removal of coronal tissue. The fracture line labially is just subgingival, and palatally it extends below the alveolar crest

restoration. ${ }^{2,5}$ It is generally accepted that there will be relapse of around $1 \mathrm{~mm}$.

\section{Case report}

A 21-year-old Caucasian male was referred to our anxiety management clinic following an alleged assault. Clinical and radiographic examination revealed an oblique root fracture in UL1 (21) in a short post and core retained extra-coronal restoration with an adequately obturated root canal. The apical extent of the fracture was apparent following the removal of the coronal portion using local anaesthetic and inhalation sedation (Fig. 1). All the remaining upper incisors were clinically and radiographically normal.

An impression was obtained for a temporary tooth bearing acrylic prosthesis to replace UL1 and a periodontal dressing placed over the root face prior to recovery from the inhalation sedation. The patient was then counselled regarding possible treatment options.

At the review appointment to fit the temporary prosthesis, and in the absence of any further symptoms, it was decided to restore the tooth with a post, core, diaphragm and crown after rapid orthodontic extrusion of the root. 


\section{PRACTICE case report}

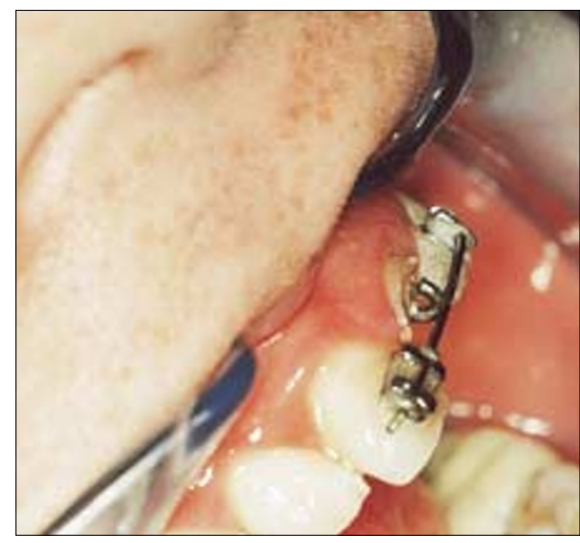

Fig. 2 Position after extrusion. Note: $3 \mathrm{~mm}$ of labial tooth tissue is now visible

At a subsequent appointment under local anaesthetic and inhalation sedation the canal orifice was enlarged and the existing gutta percha removed to within $4 \mathrm{~mm}$ of the root apex with a selection of gates-glidden burs. A small friction stop was created in the canal with an appropriate para-post drill. A piece of $1.25 \mathrm{~mm}$ diameter wiptam wire was then used to construct a temporary post. The coronal end was bent to produce a j-hook and the straight portion of the post was ridged using a diamond bur in an air rotor. The post was cemented into the root canal with IRM cement. Two edge-wise brackets were then positioned on the UR1(11) and UL2 (22) abutment teeth and cemented with dual cure composite cement. A straight rectangular stainless-steel wire $0.45 \mathrm{~mm}$ was then contoured to fit passively in the bracket slots and secured with elastic ligatures. Adequate clearance between the j-hook and the wire joining the abutment teeth was confirmed, and was crucial so that extrusion could occur. Activation was started using power chain stretched from the j-hook over the abutment wire and back to the j-hook.

After 4 weeks around $3 \mathrm{~mm}$ of extrusion had been achieved (Fig. 2). Note in this figure how the j-hook is touching the abutment wire and $3 \mathrm{~mm}$ of labial tooth tissue is visible adjacent to the labial gingival margin. Prior to the extrusion no tooth tissue was visible coronal to the gingival margin.

The position of the tooth was then retained for 6 weeks. A small amount of both labial and palatal mucosal recontouring was then necessary with electrosurgery as part of the tissue management to create a small gutter, thus allowing an accurate margin on the palatal diaphragm and on the finished crown to be produced, prior to preparation of the canal to accept a long post. The root face was also modified to receive a palatal diaphragm (Fig. 3). The cast post (Fig. 4) and the final coronal restoration (Fig. 5) were cemented with glass ionomer cement.

The patient was reviewed after 3 and 6 months and reported no problems. There were no clinical or radiographic signs of

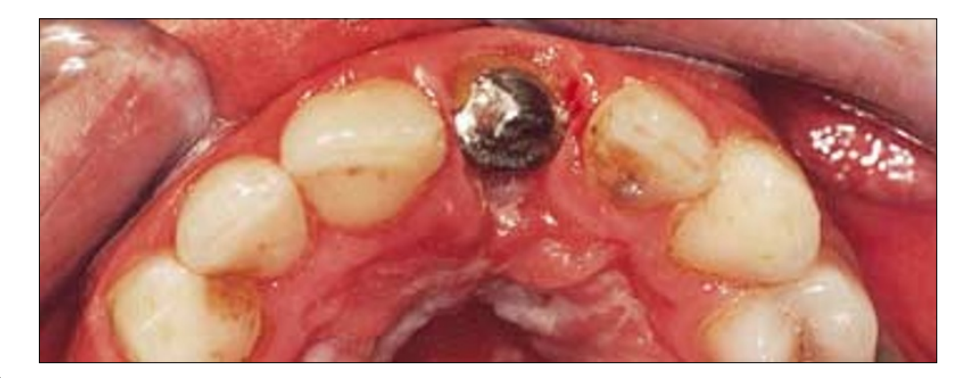
and diaphragm cemented in position

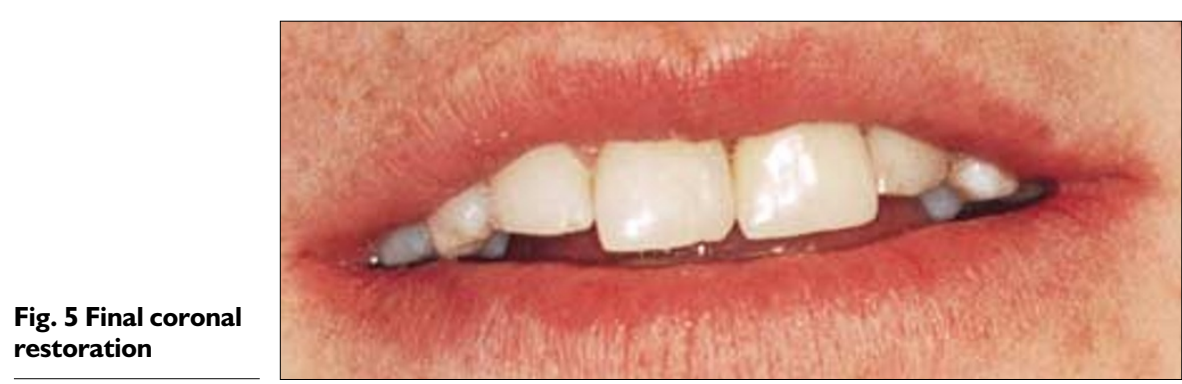

Fig. 5 Final coronal restoration infection affecting UL1 and the gingival tissues were healthy. The crown of the tooth was stable and the incisal length was unaltered. The remaining upper incisors were clinically and radiographically normal.

\section{Discussion}

The treatment described requires commitment and motivation from the patient and the dentist in order to achieve a satisfactory result. However, it is less destructive of tooth tissue than extraction and subsequent restoration with a fixed-fixed bridge or osseointegrated implant, and is more natural to a patient than an acrylic or chrome prosthesis. Prior approval should be sought by the Dental Practice Board if the treatment is to be provided under the terms of service within the National Health Service.

\section{Conclusion}

Rapid orthodontic extrusion of complicated crown root fractures involving incisors is a viable treatment option and has many clinical and financial advantages over other treatments. Currently the authors have no experience of applying this treatment modality to posterior units. However, the same principles could be applied to a single rooted posterior unit.

1 Heithersay G S. Combined endodonticorthodontic treatment of transverse root fractures in the region of the alveolar crest. Oral Surg, Oral Med, Oral Path 1973; 36: 404-415.

2 Ingber J S. Forced eruption of teeth: Part 1. A method of treating isolated one and two wall infrabony osseous defects-rationale and case report. J Periodontol 1974; 45:199-206.

3 Ingber J S. Forced eruption of teeth: Part 2. A method of treating nonrestoreable teethperiodontal and restorative considerations. $J$ Periodontol 1976; 47: 203-216.

4 Simon J H S. Root extrusion. Rationale and technique. Dent Clin N Am 1984; 28: 909-921.

5 Cook M S, Scheer B. Extrusion of fractured teeth. Br Dent J 1980; 149: 50-53.

6 Reitan K. Clinical and histological observations on tooth movements during and after orthodontic treatment. Am J Orthod 1967; 53: 721-745. 